\title{
Flow and heat transfer characteristics on a moving plate in a nanofluid
}

\begin{abstract}
This paper considers the extended Blasius and Sakiadis problems in nanofluids, by considering a uniform free stream parallel to a fixed or moving flat plate, which has more practical significance. It is assumed that the plate moves in the same or opposite direction to the free stream. The resulting system of nonlinear ordinary differential equations is solved numerically for three types of nanoparticles, namely copper $(\mathrm{Cu})$, alumina $(\mathrm{Al} 2 \mathrm{O} 3)$, and titania (TiO2) in the water based fluid with Prandtl number $\mathrm{Pr}=6.2$. The effect of the solid volume fraction parameter $\varphi$ of the nanofluids on the heat transfer characteristics is investigated. The results indicate that dual solutions exist when the plate and the free stream move in the opposite directions.
\end{abstract}

Keyword: Nanofluids; Moving plate; Heat transfer; Dual solutions 